\title{
Long non-coding RNA fer-1-like family member 4 serves as a tumor suppressor in laryngeal squamous cell carcinoma cells via regulating the AKT/ERK signaling pathway
}

\author{
LULU JIAO $^{1 *}$, SIMING LIU ${ }^{1 *}$, LILI LIU ${ }^{1}$, PENGPENG HAO ${ }^{1}$, ZHENG GONG $^{1}$, \\ ZHANFENG YAN $^{1}$ and YINZHOU XIANG ${ }^{2}$ \\ ${ }^{1}$ Department of Otorhinolaryngology, Dongzhimen Hospital, Beijing University of Chinese Medicine, Beijing 100700; \\ ${ }^{2}$ Department of Otorhinolaryngology, Taizhou First People's Hospital, Taizhou, Zhejiang 318020, P.R. China
}

Received June 10, 2020; Accepted August 26, 2020

DOI: $10.3892 / \mathrm{mmr} .2020 .11598$

\begin{abstract}
Laryngeal squamous cell carcinoma (LSCC) is a common type of malignant tumor of the head and neck. An increasing number of studies have illustrated that long non-coding RNAs (lncRNAs) serve an important role in the occurrence and development of LSCC. Therefore, the present study aimed to investigate the expression changes and mechanism of lncRNA fer-1-like family member 4 (FER1L4) in the progression of LSCC. The expression levels of FER1L4 in LSCC cell lines (AMC-HN-8, Tu 686, M4E and M2E) and a normal cell line (HBE135-E6E7) were analyzed using reverse transcription-quantitative PCR. The FER1L4 overexpression plasmid (plasmid-FER1L4) was subsequently transfected into Tu 686 cells to upregulate the expression levels of FER1L4. Cell viability was detected using a Cell Counting Kit-8 assay, cell proliferation was analyzed using a colony formation assay, apoptosis was examined by flow cytometry, and cell migration and invasion were determined using wound healing and Transwell assays, respectively. In addition, the plasmid-FER1L4 cells were also treated with insulin-like growth factor 1 (IGF-1) to determine the effect of FER1L4 on the AKT/ERK signaling pathway, and the effect of the plasmid-FER1L4 on the expression levels of AKT/ERK signaling pathway-related proteins were analyzed using western blotting. The results of the present study revealed that FER1L4 expression levels were downregulated in AMC-HN-8 and Tu 686 cells. Notably, FER1L overexpression significantly reduced the cell viability, proliferation, migration and invasion
\end{abstract}

Correspondence to: Dr Yinzhou Xiang, Department of Otorhinolaryngology, Taizhou First People's Hospital, 218 Hengjie Road, Taizhou, Zhejiang 318020, P.R. China

E-mail: yin_zhou1532@163.com

${ }^{*}$ Contributed equally

Key words: fer-1-like family member 4, laryngeal squamous cell carcinoma, AKT/ERK of LSCC cells, while promoting apoptosis. Meanwhile, the plasmid-FER1L4 also significantly suppressed the phosphorylation levels of AKT and ERK. Further studies indicated that the aforementioned changes could be reversed by IGF-1, indicating FER1L4 may regulate the progression of LSCC cells by inhibiting the AKT/ERK signaling pathway. In conclusion, the present study provided a potential novel direction for the treatment of LSCC in the future and suggested that FER1L4 may be a new target in this field.

\section{Introduction}

Laryngeal cancer is the most common type of head and neck tumor. The most common pathological type of laryngeal cancer is laryngeal squamous cell carcinoma (LSCC), accounting for $\sim 96 \%$ (1). LSCC is a highly invasive malignant tumor, which accounts for $2.4 \%$ of the newly diagnosed cases of malignancy worldwide each year $(2,3)$. The pathogenesis of the disease is associated with several factors, including the external environment, genetic factors, poor lifestyle habits and human papillomavirus infection (4,5). At present, the treatment of LSCC primarily involves surgical resection, radiotherapy and chemotherapy, which can be used to cure LSCC at early stages (6). However, due to concealed clinical manifestations of LSCC, the majority of patients are diagnosed at the middle or advanced stages, which are associated with a poor prognosis, high mortalities and serious complications $(7,8)$. The occurrence and development of LSCC are complex processes involving multiple genes and signaling pathways. For example, high expression of reversion-inducing cysteine-rich protein with Kazal motifs gene is closely related to the invasion, metastasis, pathological differentiation and clinical stages of LSCC cells (9). Cell cycle arrest induced by p21-activated kinase 4 could activate the ATM/CHEK1/CHEK2/p53 signaling pathway and inhibit the occurrence and development of LSCC cells (10). Therefore, an in-depth study into the regulation of related genes may provide a novel method for cancer diagnosis and treatment.

Commonly found in eukaryotic cells, long non-coding RNAs (lncRNAs) are a novel class of non-coding RNAs of $>200$ nucleotides in length $(11,12)$. IncRNAs can regulate 
protein-coding genes through a diverse range of mechanisms, including regulation of transcription, cell proliferation and differentiation, and they have been closely associated with the occurrence and development of numerous types of cancer, including breast, pancreatic, colorectal and gastric cancer $(13,14)$. In fact, lncRNAs have been reported to serve an important role during LSCC occurrence, development and metastasis $(15,16)$. For example, one study demonstrated that IncRNA H19 functioned as either a tumor promoter or suppressor in different tumor cells, including breast epithelial, glioblastoma and fibroblast cells (17). Luo et al (18) reported that $\mathrm{H} 19$ regulated the occurrence of LSCC through competitively binding to insulin-like growth factor (IGF)-2 and serving as a microRNA (miR) precursor that was positively related to disease progression. Li et al (19) discovered that the expression levels of HOX transcript antisense RNA (HOTAIR) were associated with the clinical stage and tumor differentiation of LSCC. In addition, upregulated expression levels of HOTAIR were associated with a lower survival rate of patients with LSCC (19). Feng et al (20) identified that metastasis associated lung adenocarcinoma transcript 1 (MALAT1) was upregulated in LSCC and the expression levels of MALAT1 were closely associated with the degree of tumor differentiation, lymph node metastasis and pathological differentiation. Fer-1-like family member 4 (FER1L4) was also identified to serve as a tumor suppressor gene in several types of tumor (21). For instance, the knockdown of FER1L4 in hepatocellular carcinoma (HCC) promoted cell proliferation and invasion (22); in colon cancer, the overexpression of FER1L4 inhibited the progression by targeting miR-106a-5p (23); in esophageal squamous cell carcinoma (ESCC), the expression levels of FER1L4 were downregulated in the ESCC tissues compared with the normal tissues; and the overexpression of FER1L4 significantly suppressed ESCC cell proliferation and migration, and induced apoptosis (24). In addition, FER1L4 demonstrated a significant inhibitory effect on various other types of cancer, including lung (25), prostate (26) and gastric cancer (27). These results indicated that the downregulated expression levels of FER1L4 may be related to the formation of numerous types of cancer, which suggests that FER1L4 has a broad research value. However, to the best of our knowledge, no study to date has reported on the expression levels and mechanism of action of FER1L4 in LSCC.

In the present study, Cell Counting Kit-8 (CCK-8), colony formation, flow cytometry, cell migration/invasion assays and western blotting were used to evaluate the effect of FER1L4 on the viability, proliferation, apoptosis, migration, invasion and the expression levels of AKT/ERK signaling pathway-related proteins, respectively, of Tu 686 cells. In addition, the mechanism of FER1L4 in LSCC was preliminarily discussed, which may provide a novel potential therapeutic target for the development of drugs for the treatment of LSCC.

\section{Materials and methods}

Cell culture. Four LSCC cell lines (AMC-HN-8, Tu 686, M4E and M2E) and one human bronchial epithelial cell line (HBE135-E6E7) were used in the present study. AMC-HN-8 (cat. no. BNCC338377) and Tu 686 (cat. no. BNCC100479) cells were obtained from the BeNa Culture Collection;
Beijing Beina Chunglian Biotechnology Research Institute. M4E (cat. no. JN-2244) and M2E (cat. no. JN-2245) cells were provided from Shanghai Jining Industrial Co., Ltd. HBE135-E6E7 cells (ATCC CRL-2741) were purchased from the American Type Culture Collection. LSCC cell lines were cultured in DMEM low glucose (Hyclone; Cytiva) supplemented with 10\% FBS (Hyclone; Cytiva) and $100 \mathrm{U} / \mathrm{ml}$ penicillin/streptomycin (Gibco; Thermo Fisher Scientific, Inc.). The HBE135-E6E7 cell line was cultured in RPMI-1640 medium (Gibco; Thermo Fisher Scientific, Inc.) supplemented with $10 \%$ FBS. All cells were cultured in a $5 \% \mathrm{CO}_{2}$ incubator at $37^{\circ} \mathrm{C}$. Cells were selected for following experiments when they were in the logarithmic phase.

Cell transfection. The FER1L4 sequence was synthesized by Shanghai GenePharma Co., Ltd., and cloned into the pcDNA3.1 vector (plasmid-FER1L4; Invitrogen; Thermo Fisher Scientific, Inc.). The corresponding empty pcDNA3.1 vector [plasmid-negative control (NC)] was used as the NC. Untransfected cells were used as the control. Tu 686 cells $\left(5 \times 10^{4}\right.$ cells/well) in the logarithmic phase were seeded into a six-well plate and these plasmids were transfected into the Tu 686 cells at a final concentration of $100 \mathrm{nM}$ using Lipofectamine ${ }^{\circledR} 2000$ reagent (Invitrogen; Thermo Fisher Scientific, Inc.). Following transfection for $48 \mathrm{~h}$ at room temperature, the transfection efficiency was analyzed using reverse transcription-quantitative PCR (RT-qPCR).

CCK-8 assay. Tu 686 cells were seeded into 96 -well plates at a density of 5,000 cells per well. Following incubation for 24, 48 and $72 \mathrm{~h}$ at $37^{\circ} \mathrm{C}, 10 \mu \mathrm{l}$ CCK-8 solution (Beijing Solarbio Science \& Technology Co., Ltd.) was added to each well and incubated in a humidified atmosphere of $95 \% \mathrm{O}_{2}$ and $5 \% \mathrm{CO}_{2}$ at $37^{\circ} \mathrm{C}$ for $2 \mathrm{~h}$, according to the manufacturer's protocols. The absorbance of each well at $450 \mathrm{~nm}$ was subsequently measured using a microplate reader (BioTek Instruments, Inc.).

Colony formation assay. Tu 686 cells were harvested $48 \mathrm{~h}$ post-transfection and incubated in six-well plates at a density of 5,000 cells per well; the medium was changed every $4 \mathrm{~h}$. Following 14 days of incubation in a humidified incubator at $37^{\circ} \mathrm{C}$ with $5 \% \mathrm{CO}_{2}$, the cells were rinsed with cold PBS, fixed with $4 \%$ methanol for $15 \mathrm{~min}$ and then stained with $0.5 \%$ crystal violet for $20 \mathrm{~min}$ at room temperature. The colonies ( $>50$ cells) were visualized under a confocal microscopy (Nikon Corporation).

Wound healing assay. Wound healing assays were used to analyze the cell migratory abilities. Briefly, Tu 686 cells were plated into six-well plates at the density of $5 \times 10^{5}$ cells $/ \mathrm{ml}$. Upon reaching $\sim 100 \%$ confluence, a $200 \mu$ l-pipette tip was used to gently scratch the adherent cell layer in the vertical direction of the well. Then, PBS was used to wash off the non-suspended cells and $100 \mu \mathrm{g} / \mathrm{ml}$ DMEM without serum was added to each well. The cells were cultured in a $\mathrm{CO}_{2}$ incubator at $37^{\circ} \mathrm{C}$ for $48 \mathrm{~h}$. The width of the wound was photographed under a light microscope (magnification, $\mathrm{x} 200$ ) and the extent of wound closure was semi-quantified by ImageJ software 2.0 (National Institutes of Health) at 0 and $48 \mathrm{~h}$. The cell migration rate was calculated as: Mobility $(\%)=(0-48 \mathrm{~h}$ scratch distance/initial distance $) \times 100 \%$. 
Invasion assay. For cell invasion experiments, $1 \times 10^{6}$ $\mathrm{Tu} 686$ cells/well were plated into the upper chamber of a Transwell plate precoated with Matrigel for $30 \mathrm{~min}$ at $37^{\circ} \mathrm{C}$ $(2 \mathrm{mg} / \mathrm{ml} ; 15 \mu \mathrm{l})$ in serum-free DMEM. DMEM $(600 \mu \mathrm{l})$ containing $10 \%$ FBS was plated into the lower chambers. Following incubation for $24 \mathrm{~h}$ at $37^{\circ} \mathrm{C}$ with $5 \% \mathrm{CO}_{2}$, the cells were stained with $0.1 \%$ crystal violet for $20 \mathrm{~min}$ at room temperature. Finally, the cells were visualized under an inverted light microscope (magnification, x100; Olympus Corporation). Invasion was semi-quantified by ImageJ software.

Cell apoptotic analysis. Flow cytometry was used to primarily analyze the proportion of cells in the late apoptosis stage. The digested cells were centrifuged at $1,000 \mathrm{x}$ g for $10 \mathrm{~min}$ at $4^{\circ} \mathrm{C}$, and the supernatant was discarded. Cold PBS was used to collect the Tu 686 cells and wash them twice. Cell apoptosis was analyzed according to the manufacturer's protocol of the Annexin V-FITC Apoptosis Detection kit (EBioscience; Thermo Fisher Scientific, Inc.). Briefly, Annexin V-FITC binding buffer was used to resuspend the Tu 686 cells, which was then diluted to provide a final concentration of $\sim 1 \times 10^{8}$ cells $/ \mathrm{ml}$. Cells were transferred to the flow tube and incubated with $5 \mu \mathrm{l}$ Annexin V-FITC staining solution for $15 \mathrm{~min}$ at in the dark at room temperature. Subsequently, the cells were incubated with $10 \mu \mathrm{l}$ propidium iodide staining solution for $5 \mathrm{~min}$ at $4^{\circ} \mathrm{C}$. Apoptotic cells were then analyzed using a flow cytometer (FC 5000; BD Biosciences) within $1 \mathrm{~h}$, and flow cytometry data were analyzed using FlowJo software v10.4.2 (FlowJo LLC).

$R T-q P C R$. Total RNA was extracted from Tu 686 cells using TRIzol $^{\circledR}$ reagent (Invitrogen; Thermo Fisher Scientific, Inc.), according to the manufacturer's protocol. Total RNA was reverse transcribed into cDNA at $42^{\circ} \mathrm{C}$ for $1 \mathrm{~h}$ using the PrimeScript RT Reagent kit (Takara Biotechnology Co., Ltd.). The following thermocycling conditions were used for RT-qPCR: $95^{\circ} \mathrm{C}$ for $5 \mathrm{sec}$; followed by 45 cycles of denaturation at $95^{\circ} \mathrm{C}$ for $20 \mathrm{sec}$, annealing at $58^{\circ} \mathrm{C}$ for $20 \mathrm{sec}$, and a final extension at $72^{\circ} \mathrm{C}$ for $30 \mathrm{sec}$. qPCR was subsequently performed using a SYBR Green PCR Master Mix (Roche Diagnostics) to analyze the expression levels of FER1L4. The following primers sequences were used: FER1L4 forward, 5'-CCGTGT TGAGGTGCTGTTC-3' and reverse, 5'-GGCAAGTCCACT GTCAGATG-3'; GAPDH forward, 5'-CAATGACCCCTTCAT TGACC-3' and reverse, 5'-GACAAGCTTCCCGTTCTCAG-3'. GAPDH was used as the internal loading control. The $2^{-\Delta \Delta C q}$ method (28) was used to quantify the mRNA expression levels.

Western blotting. Total protein was extracted from Tu 686 cells using RIPA lysis buffer (Beyotime Institute of Biotechnology). Total protein was quantified using the BCA method and $30 \mu \mathrm{g}$ protein/lane was separated via $10 \%$ SDS-PAGE. The separated proteins were subsequently transferred onto a polyvinylidene difluoride membrane and blocked with $5 \%$ skimmed milk overnight at $4^{\circ} \mathrm{C}$. The membranes were then incubated with the following primary antibodies at $4^{\circ} \mathrm{C}$ overnight: Anti-matrix metalloproteinase (MMP)-2 (1:1,000; cat. no. 40994; Cell Signaling Technology, Inc.), anti-MMP-9 (1:1,000; cat. no. 13667; Cell Signaling Technology, Inc.), anti-phosphorylated (p)-AKT (1:2,000; cat. no. 4060; Cell Signaling Technology, Inc.), anti-AKT (1:1,000; cat. no. 4691;

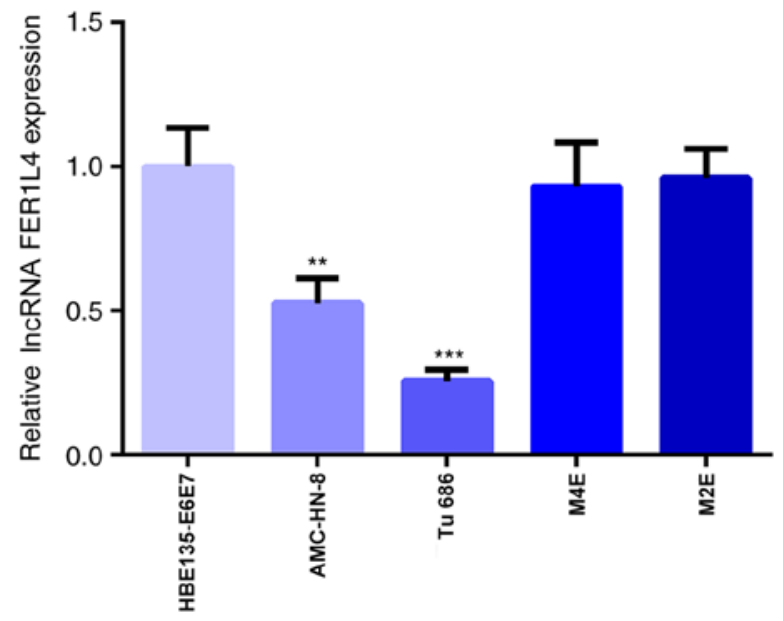

Figure 1. FER1L4 expression levels are downregulated in LSCC cell lines. Comparison of the relative expression levels of FER1L4 in the human bronchial epithelial cell line HBE135-E6E7 and human LSCC cell lines (AMC-HN-8, Tu 686, M4E and M2E). ${ }^{* *} \mathrm{P}<0.01$ and ${ }^{* * * *} \mathrm{P}<0.001$ vs. HBE135-E6E7. FER1L4, fer-1-like family member 4; lncRNA, long non-coding RNA; LSCC, laryngeal squamous cell carcinoma.

Cell Signaling Technology, Inc.), anti-p-ERK (1:2,000; cat. no. 4370; Cell Signaling Technology, Inc.), anti-ERK (1:1,000; cat. no. 4695; Cell Signaling Technology, Inc.), anti-p-p38 (1:1,000; cat. no. 4511; Cell Signaling Technology, Inc.), anti-p38 (1:1,000; cat. no. 8690; Cell Signaling Technology, Inc.), anti-p-JNK (1:2,000; cat. no. 9255; Cell Signaling Technology, Inc.), anti-JNK (1:1,000; cat. no. 9252; Cell Signaling Technology, Inc.), anti-Bcl-2 (1:1,000; cat. no. 15071; Cell Signaling Technology, Inc.), anti-Bax (1:1,000; cat. no. 2772; Cell Signaling Technology, Inc.), anti-caspase-3 (1:1,000; cat. no. 9662; Cell Signaling Technology, Inc.), anti-caspase-9 (1:1,000; cat. no. 9502; Cell Signaling Technology, Inc.) and anti-GAPDH (1:1,000; cat. no. 5174; Cell Signaling Technology, Inc.). Following the primary antibody incubation, the membranes were incubated with HRP-conjugated goat anti-rabbit secondary antibody (1:5,000; cat. no. A0208; Beyotime Institute of Biotechnology) at room temperature for $2 \mathrm{~h}$. After washing the membranes with TBS with $0.05 \%$ Tween-20, protein bands were visualized using an ECL detection kit (Cytiva) and analyzed using a Bio-Rad ChemiDoc ${ }^{\mathrm{TM}}$ MP imaging system and analyzed using Image Lab software 6.0.1 (Bio-Rad Laboratories, Inc.). GAPDH was used as the internal reference gene.

Statistical analysis. All experiments were repeated at least three times. Statistical analysis was performed using GraphPad Prism 8.0 software (GraphPad Software, Inc.) and data are expressed as the mean \pm standard deviation. The two-tailed Student's t-test or a one-way ANOVA followed by a Tukey's post hoc test were used for the analyses of the differences between groups. $\mathrm{P}<0.05$ was considered to indicate a statistically significant difference.

\section{Results}

FERIL4 expression levels are downregulated in LSCC cell lines. To investigate the role of FER1L4 in LSCC, the 
A

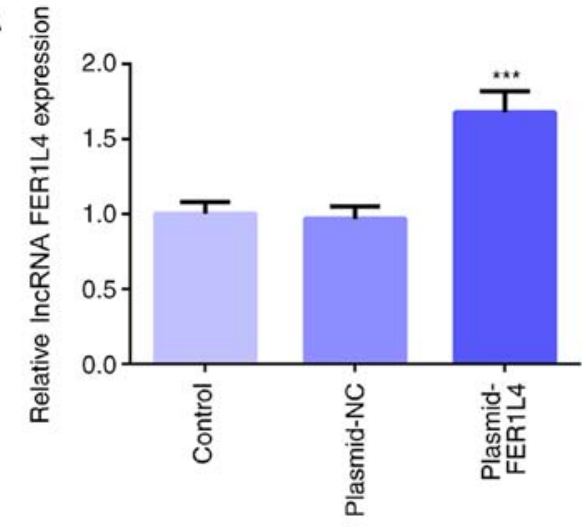

C

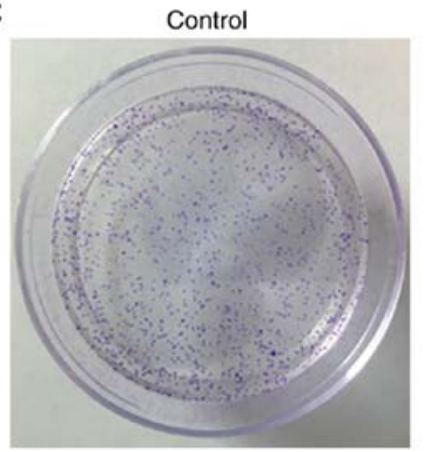

B
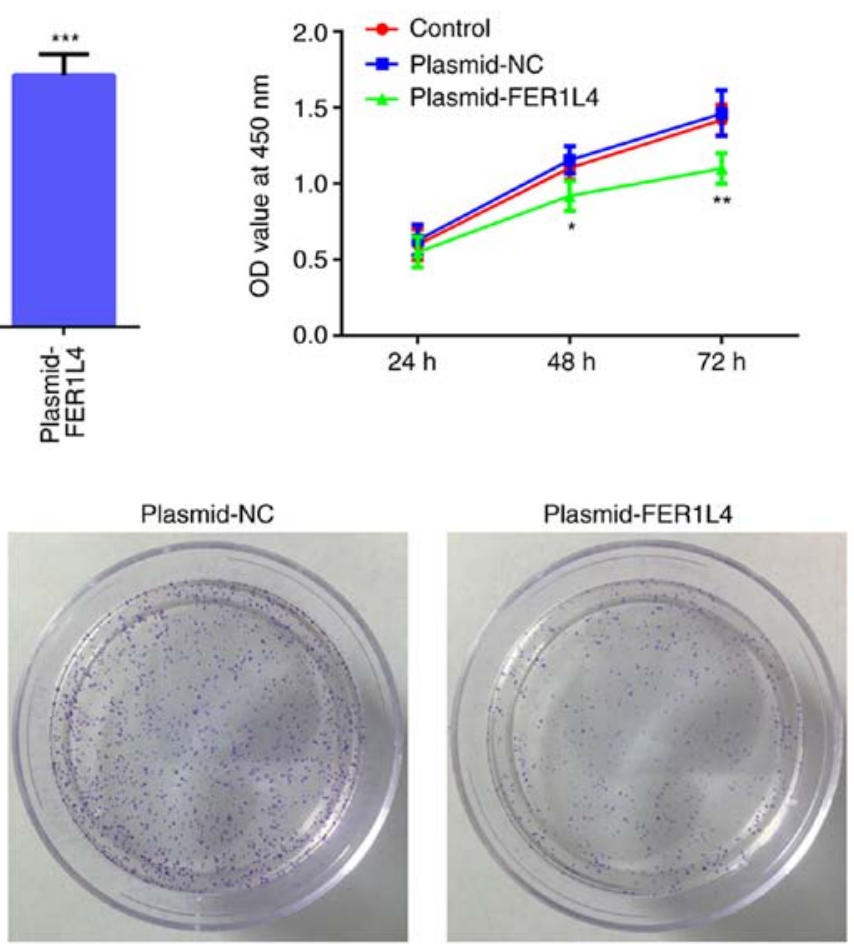

Figure 2. Overexpression of FER1L4 inhibits the cell proliferation and viability in the Tu 686 cell line. (A) Transfection efficiency of plasmid-FER1L4 was analyzed using reverse transcription-quantitative PCR. ${ }^{* * *} \mathrm{P}<0.001$ vs. control. (B) Cell Counting Kit- 8 assay was used to determine the cell viability at 24 , 48 and $72 \mathrm{~h}$. " $\mathrm{P}<0.05$ vs. control at $48 \mathrm{~h} ;{ }^{* *} \mathrm{P}<0.01$ vs. control at $72 \mathrm{~h}$. (C) Colony formation assay was used to analyze the cell proliferation ability. FER1L4, fer-1-like family member 4; lncRNA, long non-coding RNA; OD, optical density; NC, negative control.

expression levels of FER1L4 were first analyzed in different LSCC cell lines. The results of the RT-qPCR analysis are presented in Fig. 1. Compared with the HBE135-E6E7 cell line, the expression levels of FER1L4 in the LSCC cell lines, AMC-HN-8 and Tu 686, were significantly downregulated, while the expression levels of FER1L4 in the other two LSCC cell lines, M4E and M2E, were not significantly different (Fig. 1). As the expression levels of FER1L4 were the lowest in Tu 686 cells, these cells were selected for the subsequent experiments.

Overexpression of FERIL4 inhibits cell viability and proliferation in the Tu 686 cell line. The plasmid-FER1L4 or plasmid-NC were constructed and transfected into Tu 686 cells; the results demonstrated that the expression levels of FER1L4 in the plasmid-FER1L4 group were significantly upregulated compared with the control group, while there were no significant differences identified in the expression levels of FER1L4 between the plasmid-NC and control groups (Fig. 2A). The CCK-8 assay revealed that the cell viability of the plasmid-FER1L4 group was significantly decreased after $48 \mathrm{~h}$ compared with the control group (Fig. 2B). In addition, as the duration of cell culture increased, the cell viability of the plasmid-FER1L4 group was the most significantly decreased at $72 \mathrm{~h}$ compared with the control group (Fig. 2B). The colony formation assay also demonstrated that the number of colonies observed in the plasmid-FER1L4 group was decreased compared with the control and plasmid-NC groups (Fig. 2C). These results suggested that the overexpression of FER1L4 may inhibit the viability and proliferation of the Tu 686 cell line.
Overexpression of FERIL4 inhibits cell migration and invasion in the Tu 686 cell line. Wound healing and Transwell assays were used to determine the migratory and invasive ability, respectively, of Tu 686 cells. As shown in Fig. 3A and B, the results of the wound healing assay demonstrated that the overexpression of FER1L4 significantly decreased the cell migration rate and reduced the wound closure speed of Tu 686 cells compared with the control group. The results of the Transwell assay revealed that the number of invasive Tu 686 cells in the plasmid-FER1L4 group was significantly decreased compared with the control group (Fig. 3C and D). In addition, the expression levels of MMPs were investigated using western blotting. As shown in Fig. 3E, the overexpression of FER1L4 significantly downregulated the expression levels of MMP-2 and MMP-9 compared with the control group. These results suggested that the overexpression of FER1L4 may inhibit the migration and invasion of the Tu 686 cell line.

Overexpression of FERIL4 promotes apoptosis in the Tu 686 cell line. The results of the flow cytometric analysis revealed that the levels of apoptosis were significantly increased in the plasmid-FER1L4 group compared with the control group (Fig. 4A and B). The detection of apoptosis-related proteins using western blotting demonstrated that FER1L4 overexpression significantly downregulated the expression levels of Bcl-2, while it significantly upregulated the expression levels of Bax, caspase- 3 and caspase- 9 compared with the control group (Fig. 4C). These results indicated that the overexpression of FER1L4 may promote the apoptosis of Tu 686 cells. 
A

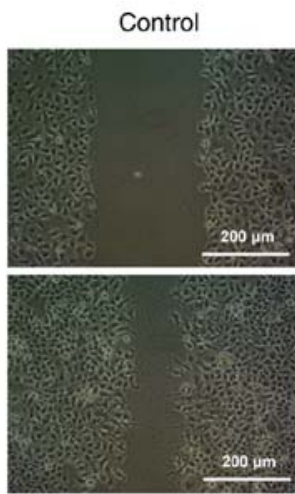

C

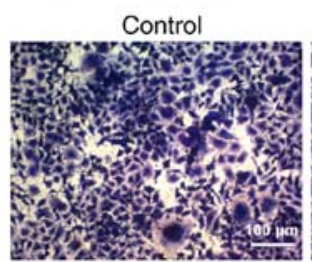

Plasmid-NC
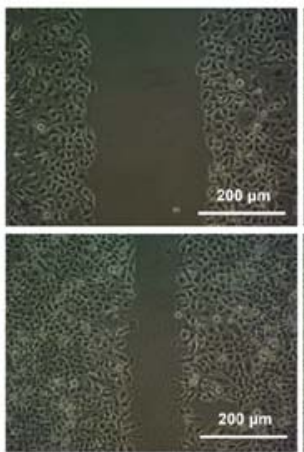

Plasmid-NC
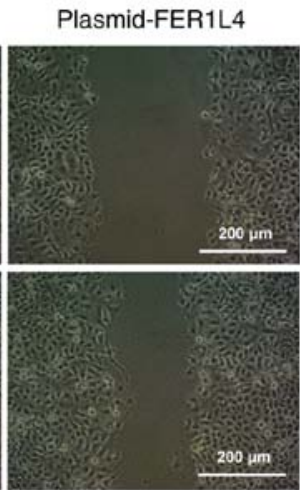

Plasmid-FER1L4

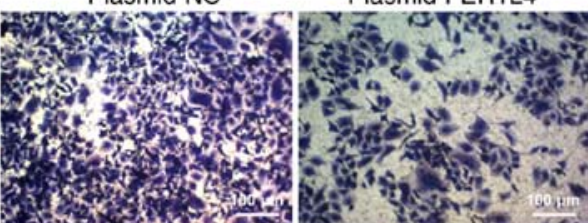

E

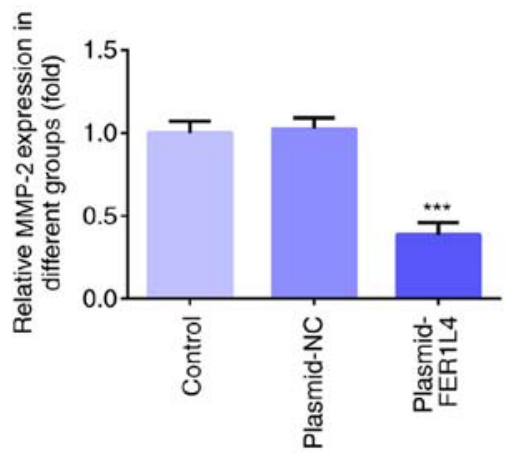

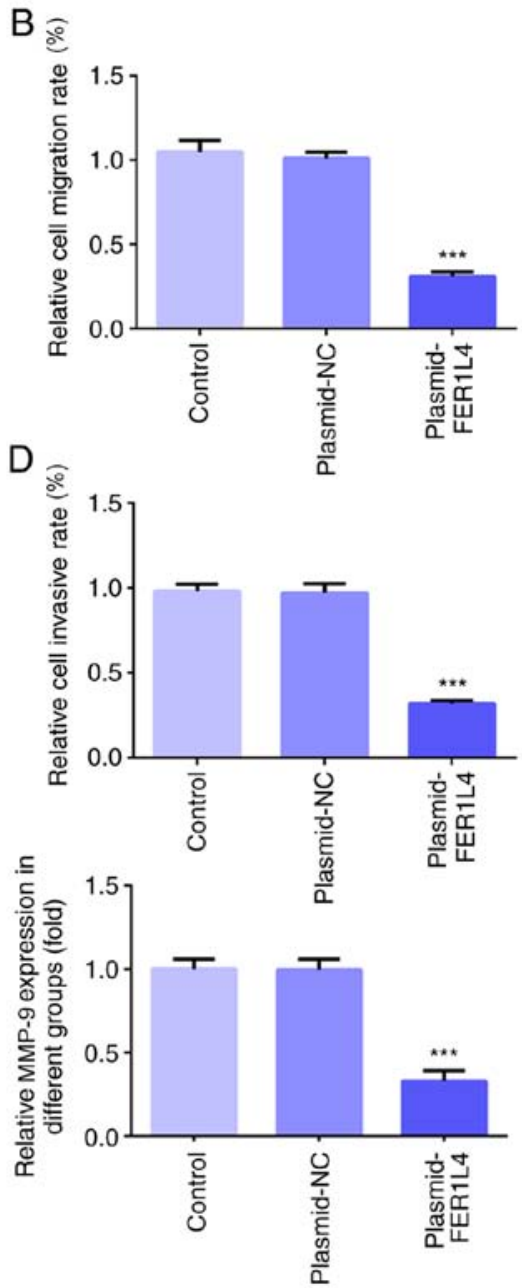

Figure 3. Overexpression of FER1L4 inhibits cell migration and invasion in the Tu 686 cell line. (A) Cell migratory ability was analyzed using a wound healing assay (scale bar, $200 \mu \mathrm{m}$ ). (B) Semi-quantification of the cell migration rate among the different groups. (C) Cell invasive ability was detected using a Transwell assay (scale bar, $100 \mu \mathrm{m}$ ). (D) Semi-quantification of the cell invasive rate among the different groups. (E) Effect of the overexpression of FER1L4 on the protein expression levels of MMP-2 and MMP-9 in Tu 686 cells was determined using western blotting. ${ }^{* * *}$ P<0.001 vs. control. FER1L4, fer-1-like family member 4; NC, negative control; MMP, matrix metalloproteinase.

FERIL4 inhibits the AKT/ERK signaling pathway in the $T u 686$ cell line. To further elucidate the regulatory mechanism of FER1L4 on the proliferation of LSCC cells, the relationship between FER1L4 and the AKT/ERK signaling pathway was investigated. Compared with the control group, the overexpression of FER1L4 significantly downregulated the expression levels of p-AKT/AKT, p-ERK/ERK, p-p38/p38 and p-JNK/JNK (Fig. 5).

Overexpression of FERIL4 inhibits cell viability and proliferation and promotes apoptosis by downregulating the AKT/ERK signaling pathway in the Tu 686 cell line. To further investigate whether FER1L4 was involved in regulating the proliferation and apoptosis of LSCC cells via the AKT/ERK signaling pathway, IGF-1, an agonist of AKT and ERK, was used to treat the transfected cells for follow-up experiments. After the plasmid-FER1L4 group was treated with IGF-1, the cell viability at all times points and the number of colonies formed increased compared with the plasmid-FER1L4 group (Fig. 6A and B). In the experiments conducted to examine cell apoptosis, the levels of apoptosis were significantly reduced in the IGF-1 + plasmid-FER1L4 group compared with the
plasmid-FER1L4 group (Fig. 6C and D). Western blotting results also demonstrated that the expression levels of Bcl-2 in the IGF-1 + plasmid-FER1L4 group were significantly upregulated, while the expression levels of Bax, caspase-3 and caspase-9 were significantly downregulated, compared with the plasmid-FER1L4 group (Fig. 6E). Based on these results, it was suggested that the overexpression of FER1L4 may inhibit the viability and proliferation, while promoting the apoptosis of Tu 686 cells through downregulating the AKT/ERK signaling pathway.

\section{Discussion}

The present study investigated the expression levels and biological function of FER1L4 in LSCC cell lines, and the findings indicated that FER1L4 expression levels in AMC-HN-8 and Tu 686 cells were significantly downregulated. In addition, the overexpression of FER1L4 significantly suppressed the cell viability, proliferation, migration and invasion, while inducing apoptosis. It was further identified that FER1L4 could inhibit the progression of LSCC by potentially modulating the AKT/ERK signaling pathway. 
A

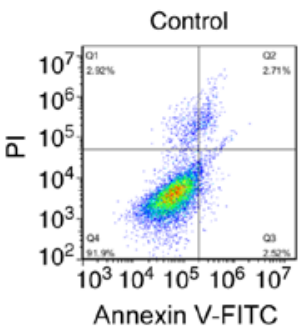

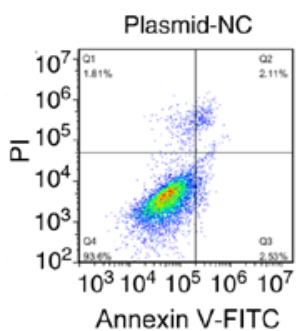
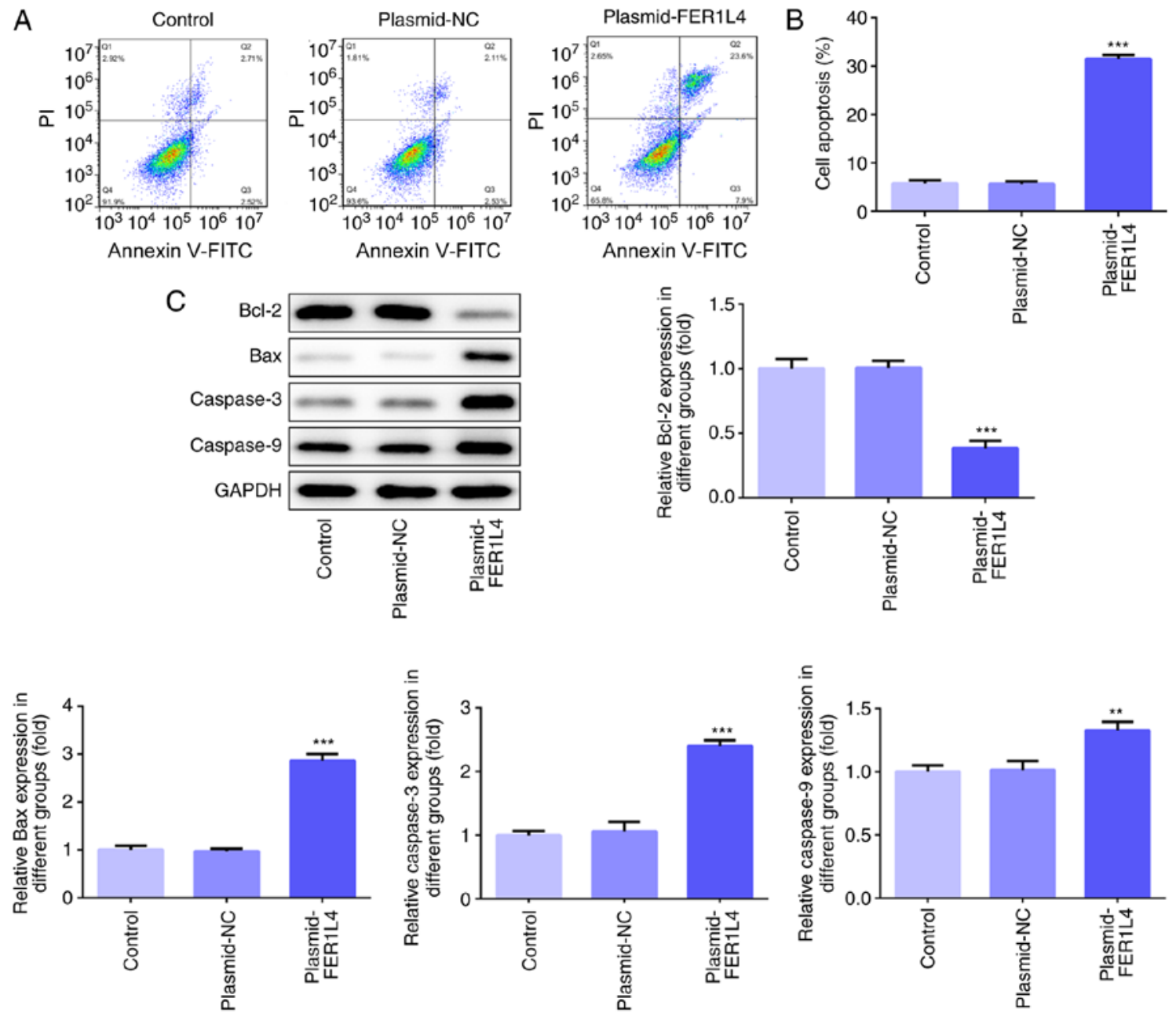

Figure 4. Overexpression of FER1L4 promotes apoptosis in the Tu 686 cell line. (A) Flow cytometric analysis of apoptosis in Tu 686 following the overexpression of FER1L4. (B) Quantification of the levels of apoptosis from part (A). (C) Effect of the overexpression of FER1L4 on the protein expression levels of Bcl-2, Bax, caspase-3 and caspase-9 in Tu 686 cells was determined using western blotting. ${ }^{* *} \mathrm{P}<0.01$ and ${ }^{* * * *} \mathrm{P}<0.001$ vs. control. FER1L4, fer-1-like family member 4; NC, negative control; PI, propidium iodide.
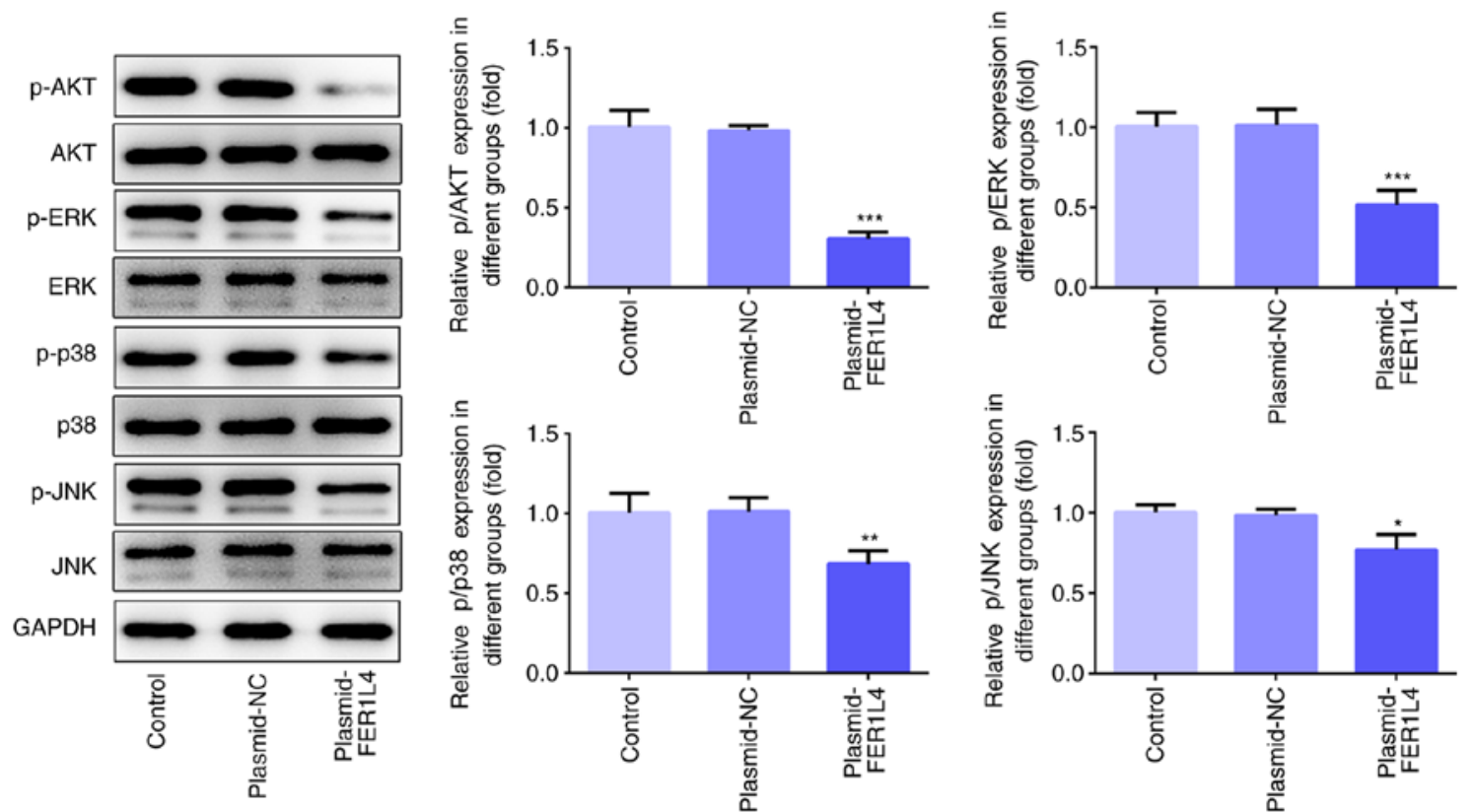

Figure 5. FER1L4 inhibits the AKT/ERK signaling pathway in the Tu 686 cell line. Effect of the overexpression of FER1L4 on the expression levels of p-AKT/AKT, p-ERK/ERK, p-p38/p38 and p-JNK/JNK. ${ }^{*} \mathrm{P}<0.05,{ }^{* * *} \mathrm{P}<0.01$ and ${ }^{* * * *} \mathrm{P}<0.001$ vs. control. FER1L4, fer-1-like family member 4; NC, negative control; p-, phosphorylated. 
A

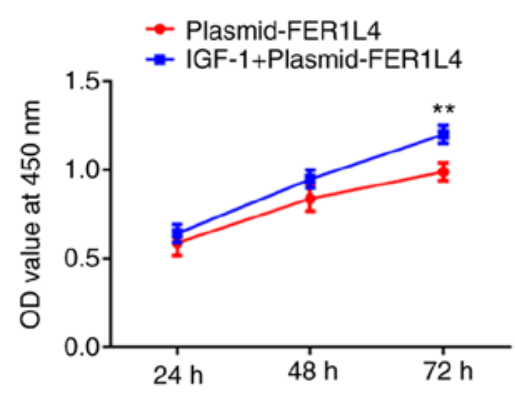

B

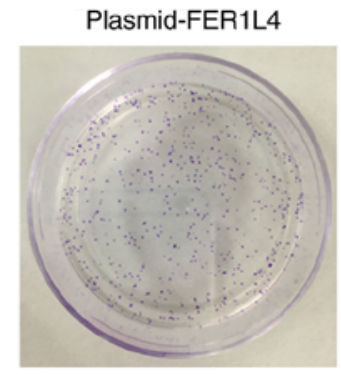

IGF-1+Plasmid-FER1L4

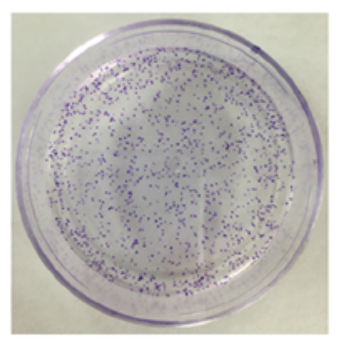

C
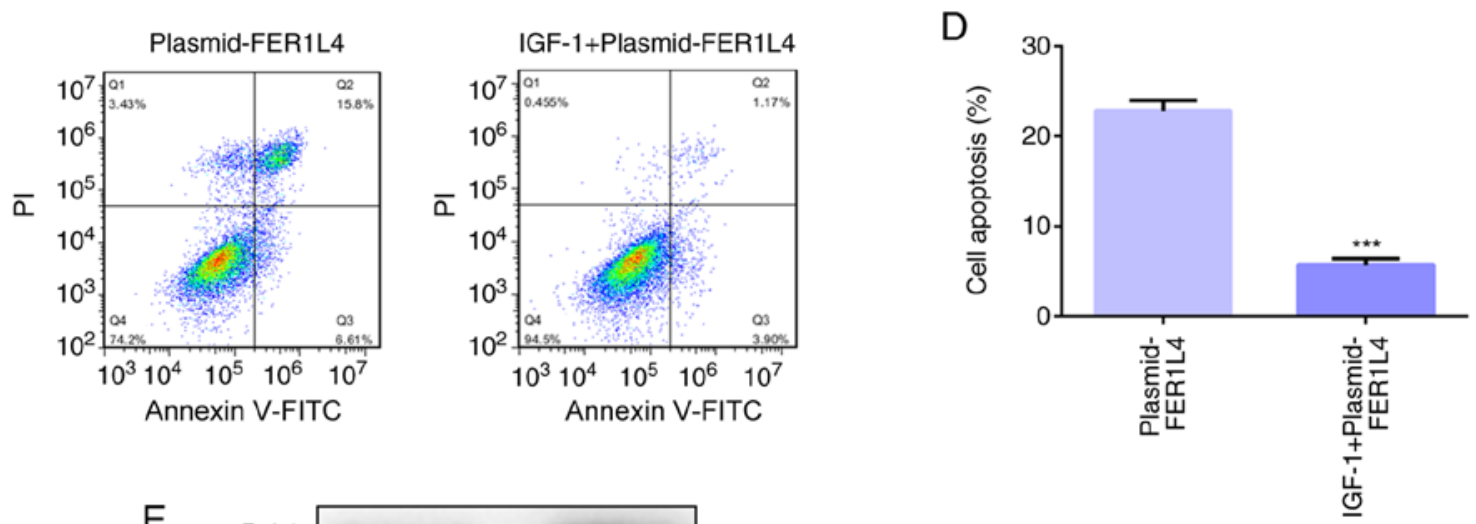

E
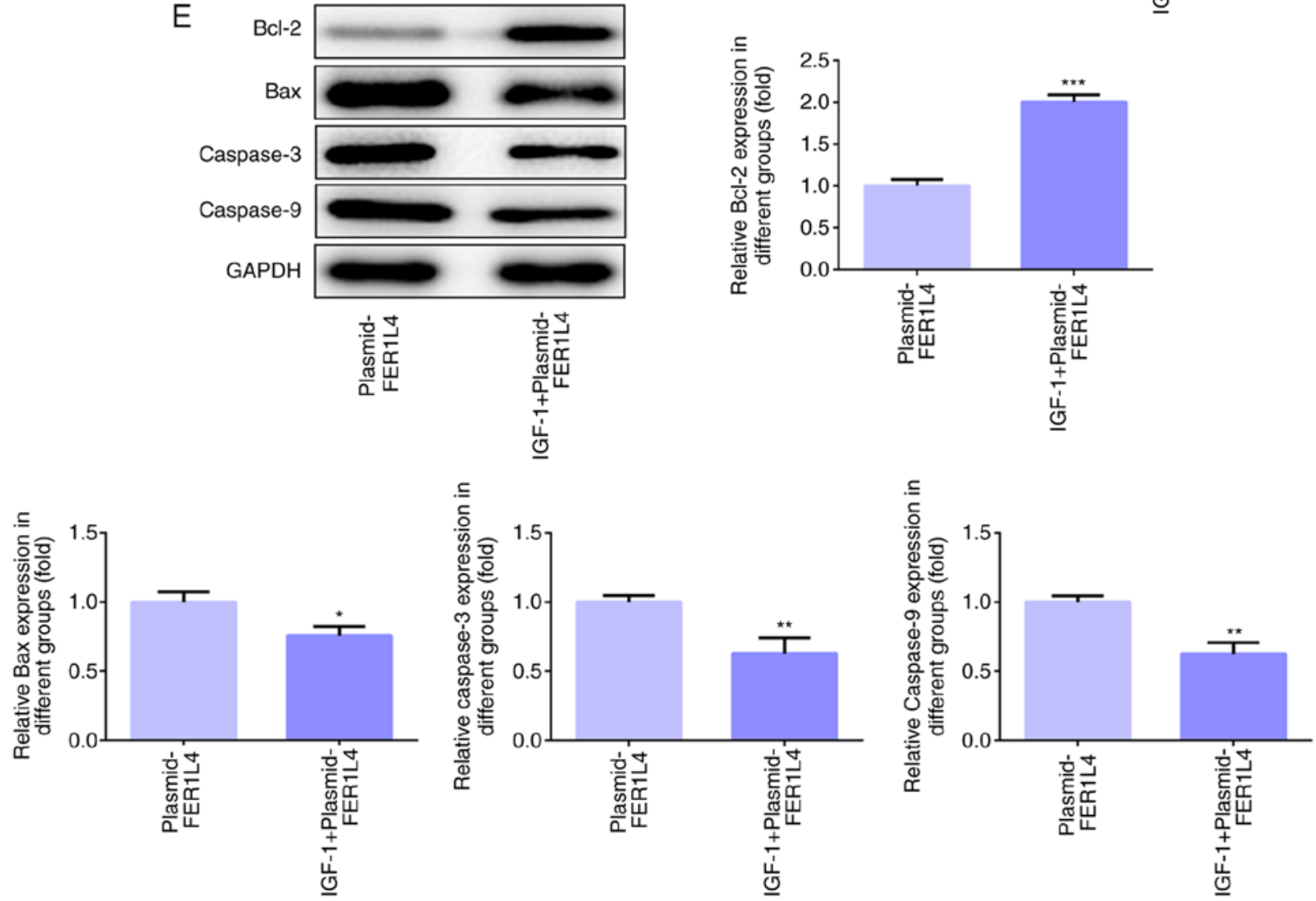

Figure 6. Overexpression of FER1L4 inhibits the cell proliferation and promotes apoptosis by downregulating the AKT/ERK signaling pathway in the Tu 686 cell line. (A) Cell Counting Kit-8 assay was used to determine the cell viability at 24, 48 and $72 \mathrm{~h}$ following the overexpression of FER1L4 in the presence or absence of IGF-1 treatment. (B) Colony formation assay was conducted to detect the cell proliferative ability following the overexpression of FER1L4 in the presence or absence of IGF-1 treatment. (C) Flow cytometric analysis of apoptosis in Tu 686 cells following the overexpression of FER1L4 in the presence or absence of IGF-1 treatment. (D) Quantification of the levels of apoptosis from part (C). (E) Effects of IGF-1 agonists on the expression levels of Bcl-2, Bax, caspase- 3 and caspase-9 in Tu 686 cells following the overexpression of FER1L4 was determined using western blotting. ${ }^{*} \mathrm{P}<0.05,{ }^{* *} \mathrm{P}<0.01$ and ${ }^{* * * *} \mathrm{P}<0.001$ vs. plasmid-FER1L4. FER1L4, fer-1-like family member 4; NC, negative control; IGF-1, insulin-like growth factor-1; PI, propidium iodide; OD, optical density.

LSCC is a malignant type of head and neck cancer (29). At present, the main clinical treatment for LSCC is surgical treatment; however, patients with LSCC often have a poor prognosis and quality of life due to the lack of effective treatment options $(30,31)$. Following increased in-depth studies at the gene level, it has been identified that abnormal gene 
expression can lead to abnormal regulatory mechanisms of tumor growth, which subsequently triggers a large number of malignant cells to proliferate, while further reducing the occurrence of apoptosis $(32,33)$. Therefore, understanding the related genes, which are associated with oncogenesis, and their effects will contribute to the prevention, treatment and prognosis of LSCC.

It has been confirmed that the abnormal expression of lncRNA is associated with the pathogenesis of a variety of types of malignant tumors, including HCC (34), pancreatic cancer (35) and colorectal cancer (36). Thus, the complex regulatory mechanism of lncRNAs has resulted in widespread interest from medical researchers $(37,38)$. FER1L4 was discovered to be expressed in a diverse range of tumors; for example, previous studies have revealed that FER1L4 inhibited the tumor cell growth, proliferation, migration and invasion of various types of cancers, including HCC, ESCC, colon cancer, lung cancer, prostate cancer and gastric cancer (22-27). In addition, FER1L4 inhibited the proliferation and migration of HCC cancer cells by regulating the P13K/AKT signaling pathway (39). However, to the best of our knowledge, the expression levels and biological function of FER1L4 in LSCC remain to be investigated. The present study demonstrated, for the first time, that the expression levels of FER1L4 were downregulated in the LSCC cell lines, AMC-HN-8 and Tu 686, in vitro. Further experimental results illustrated that the overexpression of FER1L4 significantly induced apoptosis and inhibited cell proliferation, viability, migration and invasion.

To further elucidate the molecular mechanism of FER1L4 in regulating the proliferation, apoptosis, invasion and metastasis of LSCC cells, the present study focused on the association between FER1L4 and the AKT/ERK signaling pathway in LSCC. The AKT/ERK signaling pathway is one of the most important cellular signaling pathways for cell proliferation and it has been reported to serve an important role in the occurrence and development of HCC (40), colorectal cancer (41) and ovarian cancer (42). The AKT/ERK signaling pathway has been identified to not only activate downstream signaling molecules, but also interact with other signaling molecules and result in a cascade activation $(43,44)$. However, it is not clear whether FER1L4 also affects the biological phenotype of cell proliferation, migration, invasion and apoptosis by mediating the AKT/ERK signaling pathway in LSCC. In the present study, IGF-1, an agonist of the AKT/ERK signaling pathway (45), was used to regulate the effect of FER1L4 on cell function. The present study demonstrated that FER1L4 overexpression significantly downregulated the phosphorylation levels of AKT, ERK, p38 and JNK, which are proteins related to the AKT/ERK signaling pathway (46), in Tu 686 cells, whereas IGF-1 reversed the inhibitory effects of FER1L4 overexpression on the phosphorylation levels of AKT, ERK, p38 and JNK, and alleviated the effects of FER1L4 overexpression on cell viability, proliferation and apoptosis. These results preliminarily suggested that FER1L may inhibit the proliferation, migration and invasion, while promoting the apoptosis of Tu 686 cells via inhibiting the AKT/ERK signaling pathway. However, there are a number of limitations of the present study; for example, the current study did not investigate whether there was a difference in the role of FER1L4 in vivo and in vitro, whether the downregulated expression levels of FER1L4 were related to the prognosis of patients with LSCC or how FER1L4 further affected the AKT/ERK signaling pathway through the regulation of downstream genes. Therefore, the expression levels of FER1L4 in LSCC tissues and a more detailed study of the specific effects and mechanism of FER1L4 remain to be determined in LSCC.

In conclusion, the present study demonstrated that FER1L4 overexpression inhibited the proliferation, migration and invasion, and induced apoptosis of Tu 686 cells, thus inhibiting the development of LSCC, which was indicated to potentially occur through inhibiting the AKT/ERK signaling transduction pathway. These findings suggested that FER1L4 may be a promising biological target for the gene therapy of LSCC.

\section{Acknowledgements}

Not applicable.

\section{Funding}

This research study was supported by the Beijing Natural Science Foundation (7194292), National Natural Science Foundation of China (Grants NFSC 81770993) and the Fundamental Research Funds for the Central Universities (2019-JYB-JS-052).

\section{Availability of data and materials}

The datasets used and/or analyzed during the current study are available from the corresponding author on reasonable request.

\section{Authors' contributions}

LJ, SL and YX made substantial contributions to the conception and design of the present study; LJ and SL designed the experiments; LJ, SL, LL, PH, ZG and ZY performed the experiments; LJ and SL analyzed the data and wrote the manuscript. All authors read and approved the final manuscript.

\section{Ethics approval and consent to participate}

Not applicable.

\section{Patient consent for publication}

Not applicable.

\section{Competing interests}

The authors declare that they have no competing interests.

\section{References}

1. Almadori G, Bussu F, Cadoni G, Galli J, Paludetti G and Maurizi M: Molecular markers in laryngeal squamous cell carcinoma: Towards an integrated clinicobiological approach. Eur J Cancer 41: 683-693, 2005.

2. Christensen A, Kristensen E, Therkildsen MH, Specht L, Reibel JP and Homøe P: Ten-year retrospective study of head and neck carcinoma in situ: Incidence, treatment, and clinical outcome. Oral Surg Oral Med Oral Pathol Oral Radiol 116: 174-178, 2013. 
3. Nunes Da Silva GHB, Miranda MG, Rodrigues DS, De Souza GR and Ribeiro CV: Epidemiological factors in patients with larynx cancer treated by surgery, radiotherapy or therapeutic associations. Arch Otolaryngol Rhinol 5: 43-49, 2019.

4. Edefonti V, Bravi F, Garavello W, La Vecchia C, Parpinel M, Franceschi S, Dal Maso L, Bosetti C, Boffetta P, Ferraroni M and Decarli A: Nutrient-based dietary patterns and laryngeal cancer: Evidence from an exploratory factor analysis. Cancer Epidemiol Biomarkers Prev 19: 18-27, 2010.

5. Nocini R, Molteni G, Mattiuzzi C and Lippi G: Updates on larynx cancer epidemiology. Chin J Cancer Res 32: 18-25, 2020.

6. Wu X, Cui CL, Chen WL, Fu ZY, Cui XY and Gong X: MiR-144 suppresses the growth and metastasis of laryngeal squamous cell carcinoma by targeting IRS1. Am J Transl Res 8: 1-11, 2016.

7. Forastiere AA, Goepfert H, Maor M, Pajak TF, Weber R, Morrison W, Glisson B, Trotti A, Ridge JA, Chao C, et al: Concurrent chemotherapy and radiotherapy for organ preservation in advanced laryngeal cancer. N Engl J Med 349: 2091-2098, 2003.

8. Jemal A, Bray F, Center MM, Ferlay J, Ward E and Forman D: Global cancer statistics. CA Cancer J Clin 61: 69-90, 2011.

9. Cui J, Hui LI and Linlin LU: Correlation between RECK gene methylation status and radiosensitivity in laryngeal squamous cell carcinoma. Chin J Clin Oncol 5: 315-318, 2014.

10. Sun X, Liu B, Wang J, Li J and Ji WY: Inhibition of p21-activated kinase 4 expression suppresses the proliferation of Hep-2 laryngeal carcinoma cells via activation of the ATM/Chk1/2/p53 pathway. Int J Oncol 42, 683-689, 2013.

11. Fang XY, Pan HF, Leng RX and Ye DQ: Long noncoding RNAs: Novel insights into gastric cancer. Cancer Lett 356(2 Pt B): 357-366, 2015.

12. Okazaki Y, Furuno M, Kasukawa T, Adachi J, Bono H, Kondo S, Nikaido I, Osato N, Saito R, Suzuki H, et al: Analysis of the mouse transcriptome based on functional annotation of 60,770 full-length cDNAs. Nature 420: 563-573, 2002.

13. Geisler S and Coller J: RNA in unexpected places: Long non-coding RNA functions in diverse cellular contexts. Nat Rev Mol Cell Biol 14: 699-712, 2013.

14. Jiang $\mathrm{C}$, Li X, Zhao $\mathrm{H}$ and Liu H: Long non-coding RNAs: Potential new biomarkers for predicting tumor invasion and metastasis. Mol Cancer 15: 62, 2016.

15. Wang KC, Yang YW, Liu B, Sanyal A, Corces-Zimmerman R, Chen Y, Lajoie BR, Protacio A, Flynn RA, Gupta RA, et al: A long noncoding RNA maintains active chromatin to coordinate homeotic gene expression. Nature 472: 120-124, 2011

16. Yang QQ and Deng YF: Long non-coding RNAs as novel biomarkers and therapeutic targets in head and neck cancers. In J Clin Exp Pathol 7: 1286-1292, 2014.

17. Barsyte-Lovejoy D, Lau SK, Boutros PC, Khosravi F, Jurisica I, Andrulis IL, Tsao MS and Penn LZ: The c-Myc oncogene directly induces the H19 noncoding RNA by allele-specific binding to potentiate tumorigenesis. Cancer Res 66: 5330-5337, 2006.

18. Luo H, Sun Y, Wei G, Luo J, Yang X, Liu W, Guo M and Chen R: Functional characterization of long noncoding RNA Lnc_bc060912 in human lung carcinoma cells. Biochemistry 54: 2895-2902, 2015.

19. Li D, Feng J, Wu T, Wang Y, Sun Y, Ren J and Liu M: Long intergenic noncoding RNA HOTAIR is overexpressed and regulates PTEN methylation in laryngeal squamous cell carcinoma. Am J Pathol 182: 64-70, 2013.

20. Feng J, Tian L, Sun Y, Li D, Wu T, Wang Y and Liu M: Expression of long non-coding ribonucleic acid metastasis-associated lung adenocarcinoma transcript-1 is correlated with progress and apoptosis of laryngeal squamous cell carcinoma. Head Neck Oncol 4: 46, 2012.

21. Xia T, Chen S, Jiang Z, Shao Y, Jiang X, Li P, Xiao B and Guo J: Long noncoding RNA FER1L4 suppresses cancer cell growth by acting as a competing endogenous RNA and regulating PTEN expression. Sci Rep 5: 13445, 2015.

22. Wu J, Huang J, Wang W, Xu J, Yin M, Cheng N and Yin J: Long non-coding RNA Fer-1-like protein 4 acts as a tumor suppressor via miR-106a-5p and predicts good prognosis in hepatocellular carcinoma. Cancer Biomark 20: 55-65, 2017.

23. Yue B, Sun B, Liu C, Zhao S, Zhang D, Yu F and Yan D: Long non-coding RNA Fer-1-like protein 4 suppresses oncogenesis and exhibits prognostic value by associating with miR-106a-5p in colon cancer. Cancer Sci 106: 1323-1332, 2015.

24. Ma W, Zhang CQ, Li HL, Gu J, Miao GY, Cai HY, Wang JK, Zhang LJ, Song YM, Tian YH and Song YH: LncRNA FER1L4 suppressed cancer cell growth and invasion in esophageal squamous cell carcinoma. Eur Rev Med Pharmacol Sci 22 2638-2645, 2018.
25. Gao X, Wang N, Wu S, Cui H, An X and Yang Y: Long non-coding RNA FER1L4 inhibits cell proliferation and metastasis through regulation of the PI3K/AKT signaling pathway in lung cancer cells. Mol Med Rep 20: 182-190, 2019.

26. Huo W, Qi F and Wang K: Long non-coding RNA FER1L4 inhibits prostate cancer progression via sponging miR-92a-3p and upregulation of FBXW7. Cancer Cell Int 20: 64, 2020.

27. Sun W, Yang Y, Xu C, Xie Y and Guo J: Roles of long noncoding RNAs in gastric cancer and their clinical applications. J Cancer Res Clin Oncol 142: 2231-2237, 2016.

28. Livak KJ and Schmittgen TD: Analysis of relative gene expression data using real-time quantitative PCR and the 2(-Delta Delta C(T)) method. Methods 25: 402-408, 2001.

29. Zhang W, Yan Y, Gu M, Wang X, Zhu H, Zhang S and Wang W: High expression levels of Wnt5a and Ror 2 in laryngeal squamous cell carcinoma are associated with poor prognosis. Oncol Lett 14: 2232-2238, 2017

30. Ilm K, Fuchs S, Mudduluru G and Stein U: MACC1 is post-transcriptionally regulated by miR-218 in colorectal cancer. Oncotarget 7: 53443-53458, 2016.

31. Wu Y, Jia Z, Cao D, Wang C, Wu X, You L, Wen S, Pan Y, Cao X and Jiang J: Predictive value of miR-219-1, miR-938, miR-34b/c, and miR-218 polymorphisms for gastric cancer susceptibility and prognosis. Dis Markers 2017: 4731891, 2017.

32. Si L, Zheng L, Xu L, Yin L, Han X, Qi Y, Xu Y, Wang C and Peng J: Dioscin suppresses human laryngeal cancer cells growth via induction of cell-cycle arrest and MAPK-mediated mitochondrial-derived apoptosis and inhibition of tumor invasion. Eur J Pharmacol 774: 105-117, 2016.

33. Morlando M and Fatica A: Alteration of epigenetic regulation by long noncoding RNAs in cancer. Int J Mol Sci 19: 570, 2018.

34. Wang Y, Liu Z, Yao B, Li Q, Wang L, Wang C, Dou C, Xu M, Liu Q and Tu K: Long non-coding RNA CASC2 suppresses epithelial-mesenchymal transition of hepatocellular carcinoma cells through CASC2/miR-367/FBXW7 axis. Mol Cancer 16: $123,2017$.

35. Ding N, Wu H, Tao T and Peng E: NEAT1 regulates cell proliferation and apoptosis of ovarian cancer by miR-34a-5p/BCL2 . Onco Targets Ther 10: 4905-4915, 2017

36. Peng W, Wang Z and Fan H: LncRNA NEAT1 impacts cell proliferation and apoptosis of colorectal cancer via regulation of Akt signaling. Pathol Oncol Res 23: 651-656, 2017.

37. Esteller M: Non-coding RNAs in human disease. Nat Rev Genet 12: 861-874, 2011.

38. Mercer TR, Dinger ME and Mattick JS: Long non-coding RNAs: Insights into functions. Nat Rev Genet 10: 155-159, 2009.

39. Liu S, Zou B, Tian T, Luo X, Mao B, Zhang X and Lei H: Overexpression of the lncRNA FER1L4 inhibits paclitaxel tolerance of ovarian cancer cells via the regulation of the MAPK signaling pathway. J Cell Biochem 2018 (Epub ahead of print).

40. Chen Z, Ma Y, Pan Y, Zuo S, Zhu H, Yu C, Zhu C and Sun C: Long noncoding RNA RP5-833A20. 1 suppresses tumorigenesis in hepatocellular carcinoma through Akt/ERK pathway by targeting miR-18a-5p. Onco Targets Ther 12: 10717-10726, 2019.

41. Ye Q, Cai W, Zheng Y, Evers BM and She QB: ERK and AKT signaling cooperate to translationally regulate survivin expression for metastatic progression of colorectal cancer. Oncogene 33: 1828-1839, 2014.

42. Yao N, Sun JQ, Yu L, Ma L and Guo BQ: LINC00968 accelerates the progression of epithelial ovarian cancer via mediating the cell cycle progression. Eur Rev Med Pharmacol Sci 23: 4642-4649, 2019.

43. Chang C, Xie J, Yang Q, Yang J, Luo Y, Xi L, Guo J, Yang G, Jin W and Wang G: Serine peptidase inhibitor Kazal type III (SPINK3) promotes BRL-3A cell proliferation by targeting the PI3K-AKT signaling pathway. J Cell Physiol 235: 2209-2219, 2020.

44. Meng XP, Ma J, Wang B, Wu X and Liu Z: Long non-coding RNA OIP5-AS1 promotes pancreatic cancer cell growth through sponging miR-342-3p via AKT/ERK signaling pathway. J Physiol Biochem 76: 301-315, 2020

45. Liu X, Li J and Li X: MiR-142-5p regulates the progression of diabetic retinopathy by targeting IGF1. Int J Immunopathol Pharmacol 34: 2058738420909041, 2020.

46. Cordero-Herrera I, Martín MA, Bravo L, Goya L and Ramos S: Epicatechin gallate induces cell death via p53 activation and stimulation of $\mathrm{p} 38$ and JNK in human colon cancer SW480 cells. Nutr Cancer 65: 718-728, 2013.

This work is licensed under a Creative Commons Attribution-NonCommercial-NoDerivatives 4.0 International (CC BY-NC-ND 4.0) License. 